eignet sich das Wasser der Melusinenquelle ganz besonders zu Trinkkuren, sowie zur Bereitung kohlensaurer Bäder.

Grossherzogl. Lebensmittel - Prüfungsstation Karlsruhe, im Juli 1891.

\section{Beiträge zur Analyse des Zuckers und Tannins im Wein.}

$$
\text { Von }
$$

Dr. J. H. Vogel.

$\mathrm{Zu}$ meiner kürzlich (S. 44 d. Z.) unter obigem Titel gegebenen Abhandlung bemerkt Arthur Bornträger (S. 340 d. Z.), dass bei einer von ihm angegebenen Anleitung (d. Z. 1889, 341) die Entfärbung tief gefärbter Rothweine durch Bleiessig weit rascher und besser von statten geht, als in der von mir befolgten Weise nach Vorschrift der Berliner Weincommission. Ich habe diese Vorschrift mit dem in meiner $\mathrm{Ab}$ handlung (S. 45 d. Z.) erwähnten tiefgefärbten Rothwein von Bairrada geprüft und kann allerdings bestätigen, dass nach derselben die Entfärbung rascher und besser von statten geht, als bei directer Anwendung von Bleiacetat.

Ich fand seinerzeit in dem Weine von Bairrada 0,16 Proc. Zucker. Zur Entfärbung desselben mittels Bleiessig waren auf $60 \mathrm{cc}$ des im Verbültniss von $1: 5$ verdünnten Weines 30 cc Bleiessig nöthig gewesen, $d . h$. auf 60 cc Wein 180 ce Bleiessig. Nach Bornträger gebrauchte ich nur etwas mehr als $1 / 3$ derselben Bleiessiglösung, um vollständige Entfärbung zu erzielen. Die Zuckerbestimmung in dem so entfärbten Weine ergab 0,17 Proc.

Nachdem ich indessen festgestellt habe, dass bei Anwendung von Thierkohle in der von mir angegebenen Weise keine Fehler entstehen, welche die erlaubte Grenze überschreiten, glaube ich nach wie vor diese Art der Entfärbung stark gefärbter Rothweine statt der Bornträger'schen mittels Bleiessig empfehlen zu könuen. Hier ist nur die einfache Mischung des Weines mit Koble, gelegentliches Umrübren und darauffolgende Filtration nöthig, während in jenem Falle die vorbereitenden Arbeiten sehr viel umständlicher sind, als Abmessen des Weines, Neutralisation, Einengen auf dem Wasserbade mit Controlirung der Reaction, Überspülen in einen Messkolben, Fällung mit Bleiessig und Auffüllen bis zur Marke, Filtration, sorgfältige Controle der Reaction u. s. w.
Man wird diese Mehrarbeit besonders dann sehr unangenehm empfinden, wenn man grössere Mengen von Proben gleichzeitig zur Untersuchung erhält, wie das in dem von mir geleiteten Laboratorium hier häufiger der Fall ist. Herr Bornträger dürfte bei gleichzeitiger Anstellung einer grösseren Reihe von Versuchen nach beiden Methoden mit tief gefärbten Rothweinen sich alsbald von der Zweckmässigkeit der Anwendung von Thierkohle überzeugen und stelle ich ihm, wie jedem anderen Interessenten, eine Probe derwohl schwer zu erlangenden abnorm tief gefärbten Rothweine von Bairrada auf Wunsch gern zur Verfügung.

Bei dieser Gelegenheit möge hier noch die Bemerkung Platz finden, dass Herr Franz Kathreiner, Chemiker in Worms, mich durch Zuschrift vom 9. Febr. gütigst darauf aufmerksam gemacht hat, dass er bei Gelegenheit der Beantwortung der Frage "Welche Methode der Gerbstoffbestimmung ist zur allgemeinen Annahme in der Lederfabrikation zu empfehlen?" die Carpené' sche Methode der Gerbstoffbestimmung einer eingehenden Prüfung unterzogen, welche die vollständige Unbrauchbarkeit der Methode dargethan habe. Aus der mir übersandten Originalarbeit (Dingler 227 S. 481) geht in derselben unzweifelhaften Weise, wie aus den von mir angestellten Versuchen, hervor, dass genaue Resultate mit der Methode nicht zu erzielen sind.

Coimbra, im Juni 1891.

\section{Wasserstrahl-Luftpumpe. \\ Von}

\section{Max Stuhl.}

Der Apparat arbeitet schon mit einem Wasserdruck von $1 \mathrm{Atm}$. und ist der Wasserverbrauch bedeutend geringer als bei den bisher gebräuchlichen Wasserstrahlgebläsen.

Die Handhabung des Apparates ist die denkbar einfachste, was durch Hinweglassung aller Regulirungshähne und Injectoren bedingt ist. Die Verbindung mit der Wasserzuleitung wird durch Kautschukschlauch bewirkt. Will man den Apparat zum Blasen

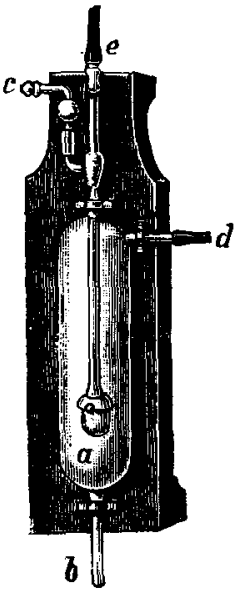

Fig. 182. 
herrichten, so verbindet man das Luftausströmungsrobr $d$ mittels Kautschukschlauch mit einer Gebläselampe und öffnet nun langsam den Wasserzuleitungshahn soweit, bis der Luftstrom die gewünschte Stärke erreicht hat und der Luftsammelraum $a$ noch wasserfrei bleibt.

Soll der Apparat zum Evacuiren benutzt werden, dann entfernt man den Kautschukschlauch von $d$ und verbindet ihn mit dem Saugrohr $c$ und den $z u$ evacuirenden Gefässen, dann schliesst man das Luftausströmungsrohr $d$ mittels der beigegebenen Gummikappe und öffnet langsam den Wasserbahn und erreicht in wenigen Secunden ein annäherndes Vacuum.

Der Apparat zeichnet sich durch seine Einfachheit und seinen äusserst kräftig wirkenden Luftstrom und eine Saugekraft bis auf $2 \mathrm{~mm}$ vor allen anderen Constructionen besonders aus.

Eine Verstopfung der Pumpe ist vollständig ausgeschlossen und seine Thätigkeit genau zu beobachten, da alle inneren Theile aus Glas und deutlich sichtbar sind.

Aus einem Stück gearbeitet und der grösseren Sicherheit wegen auf polirtem Brett befestigt, ist der Apparat von der Firma Max Stuhl, Berlin N.W. Philippstr. 21 für 13,50 Mark zu beziehen.

\section{Zur gasvolumetrischen Analyse.}

Von

\section{A. Baumann.}

Aus dem Laboratorium von Prof. Lunge theilt L. Marchlewski S. 394 d. Z. einige Analysen mit, welche er zur Prüfung der von mir beschriebenen Methoden ausgeführt hat. Da M. Resultate erhält, welche mit den theoretischen Werthen nicht übereinstimmen, so glaubt er den Schluss zieben zu dürfen, dass die Methoden ungenau und unbrauchbar seien.

Ich habe dem gegenüber nur noch zu erwidern, dass die Anzahl der Analysen, welche im hiesigen Laboratorium ausgeführt worden sind, mehr als $10 \mathrm{mal}$ so gross ist als die Anzahl der Versuche von M., und dass diese 500 bis 600 Analysen vorzügliche Resultate geliefert habcn. Es werden nun diese sämmtlichen Versuche zur Veröffentlichung gebracht in mehreren umfangreichen Abhandlungen von H. Lübcke "Über die gasvolumetrische Bestimmung der Chromsäure, des Chromoxyds und der Schwefelsäure“" und von G. Hauck "Über die gasvolumetrische Bestimmung der Verbindungen des Bleies, Wismuths und Baryums." In gleicher Weise werden die jodometrischen Methoden in mehreren besonderen Abhandlungen näber dargestellt und weiter ausgebaut werden. Sämmtliche Schriften werden mit den nöthigen Specialtabellen zum bequemen Gebrauch der Methoden ausgestattet und durch den Buchhandel zugänglich gemacht.

Hinsichtlich der Ausführung der gasvolumetrischen Methoden mittels Wasserstoffsuperoxyd möchte ich noch darauf aufmerksam machen, dass leicht zu hohe Resultate erzielt werden, wenn die Wasserstoffsuperoxydlösung in verschlossenen Gefässen aufbewahrt und vor dem jeweiligen Gebrauch nicht umgeschüttelt wird.

Denn da diese Substanz auch bei gewöhnlicher Temperatur eine fortwährende allmähliche Zersetzung unter Sauerstoffabspaltung erleidet, bleibt stets eine nicht unbeträchtliche Grasmenge in Lösung, die dann durch das heftige Schütteln bei der Bestimmung entweicht und die Resultate erhöht. Diese Gasmenge ist natürlich um so grösser, je höher der Druck ist, dem der frei werdende Sauerstoff durch den Verschluss der Flasche ausgesetzt wird; und man kann sich leicht überzeugen, wenn man solche Wasserstoffsuperoxydlösung für sich im Azotometer schüttelt, dass aus 10 cc 0,5 bis $2 \mathrm{mg}$ Sauerstoff hierdurch in Freiheit gesetzt werden können.

Bei allen Wasserstoffsuperoxydmethoden ist ferner zu beachten, dass die Lösung nicht zu concentrirt sei, weil sich sonst gleichfalls die Resultate durch Zersetzung des Wasserstoffsuperoxyds erhöhen. Man benütze eine $1^{1 / 2}$ bis 2 proc. Lösung, wie ich sie empfohlen habe und wie sie auch in der Regel im Handel vorkommt. $\mathrm{Zu}$ hohe Resultate erhält man häufig auch, wenn man einen allzu grossen Überschuss des Reagens verwendet. Deshalb werden bei unseren Bestimmungen bei Entwicklung einer Gasmenge bis $50 \mathrm{cc} 5 \mathrm{cc}$ der Superoxydlösung, falls man 50 bis 100 ce Gas zu erwarten hat, 10 cc verwendet. Die Nichtbeachtung der Verhältnisse dürfte zur Genüge die zu hohen Resultate erklären, welche Marchlewski mit unseren Methoden erbalten hat.

Was die gasvolumetrische Bestimmung des Jods betrifft, so habe ich das grösste Gewicht auf Erlangung einerkleinen manuellen Fertigkeit gelegt, welche darin besteht, dass man die Jodlösung in rasche drehende Bewegung versetzt und, ohne dieselbe zu unterbrechen, plötzlich mit sehr stark alkalischem Wasserstoffsuperoxyd mischt. Ich muss an- 